Report Title:

\title{
Carbon Ionic Conductors for use in Novel Carbon-Ion Fuel Cells
}

\author{
Type of Report:
}

Final Technical Report

Reporting Period Start Date: September 1, 2003

Reporting Period End Date: August 30, 2005

Principal Author: Franklin H. Cocks

with W. Neal Simmons and Paul A. Klenk

Date Report Issued: November, 2005

DOE Award Number: DE-FG26-03NT41804

Name and Address of Submitting Organization:

\author{
Duke University \\ Pratt School of Engineering \\ Department of Mechanical Engineering and Materials Science \\ Durham, NC 27708-0300
}




\section{DISCLAIMER}

This report was prepared as an account of work sponsored by an agency of the United States Government. Neither the United States Government nor any agency thereof, nor any of their employees, makes any warranty, express or implied, or assumes any legal liability or responsibility for the accuracy, completeness, or usefulness of any information, apparatus, product, or process disclosed, or represents that its use would not infringe privately owned rights. Reference herein to any specific commercial product, process, or service by trade name, trademark, manufacturer, or otherwise does not necessarily constitute or imply its endorsement, recommendation, or favoring by the United States Government or any agency thereof. The views and opinions of authors expressed herein do not necessarily state or reflect those of the United States Government or any agency thereof. 


\begin{abstract}
Carbon-consuming fuel cells have many potential advantages, including increased efficiency and reduced pollution in power generation from coal. A large amount of work has already been done on coal fuel cells that utilize yttria-stabilized zirconium carbide as an oxygen-ion superionic membrane material. But high-temperature fuel cells utilizing yttria-stabilized zirconium require partial combustion of coal to carbon monoxide before final oxidation to carbon dioxide occurs via utilization of the oxygen-ion zirconia membrane. A carbon-ion superionic membrane material would enable an entirely new class of carbon fuel cell to be developed, one that would use coal directly as the fuel source, without any intervening combustion process. However, a superionic membrane material for carbon ions has not yet been found. Because no partial combustion of coal would be required, a carbon-ion superionic conductor would allow the direct conversion of coal to electricity and pure $\mathrm{CO}_{2}$ without the formation of gaseous pollutants. The objective of this research was to investigate ionic lanthanide carbides, which have an unusually high carbon-bond ionicity as potential superionic carbide-ion conductors. A first step in this process is the stabilization of these carbides in the cubic structure, and this stabilization has been achieved via the preparation of pseudobinary lanthanide carbides. The diffusion rates of carbon have been measured in these carbides as stabilized to preserve the high temperature cubic structure down to room temperature. To prepare these new compounds and measure these diffusion rates, a novel, oxide-based preparation method and a new $\mathrm{C}^{13} / \mathrm{C}^{12}$ diffusion technique have been developed. The carbon diffusion rates in $\mathrm{La}_{0.5} \mathrm{Er}_{0.5} \mathrm{C}_{2}, \mathrm{Ce}_{0.5} \mathrm{Er}_{0.5} \mathrm{C}_{2}$, and $\mathrm{La}_{0.5} \mathrm{Y}_{0.5} \mathrm{C}_{2}$, and $\mathrm{Ce}_{0.5} \mathrm{Tm}_{0.5} \mathrm{C}_{2}$ modified by the addition of $5 \mathrm{wt} \% \mathrm{Be}_{2} \mathrm{C}$, have been determined at temperatures from $850^{\circ} \mathrm{C}$ to $1150^{\circ} \mathrm{C}$. The resulting diffusion constants as measured were all less than $10^{-11} \mathrm{~cm}^{2} / \mathrm{sec}$, and therefore these compounds are not superionic. However, there remain a large number of potentially superionic pseudobinary lanthanide compounds and a number of alternate ionic carbides which might act as dopants to produce vacancies on the carbonion sublattice and thereby increase carbon-ion diffusion rates. The discovery of a superionic carbon conductor would usher in a truly revolutionary new coal technology, and could dramatically improve the way in which we generate electricity from coal. The work completed to date is a promising first step towards this end.
\end{abstract}


TABLE OF CONTENTS

LIST(S) OF GRAPHICAL MATERIALS ..................................4

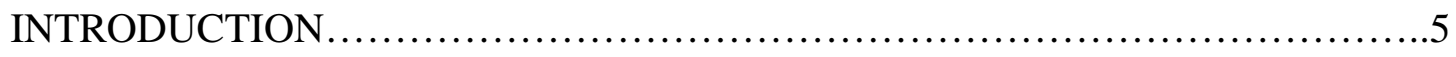

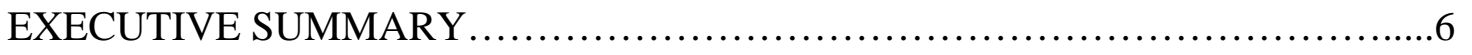

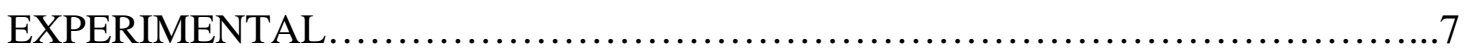

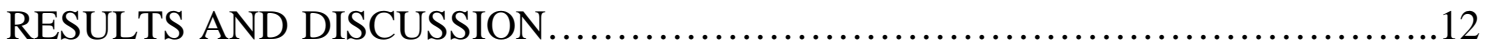

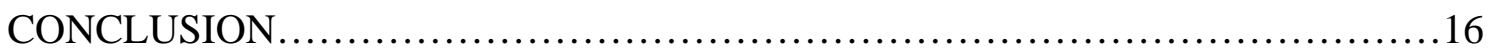

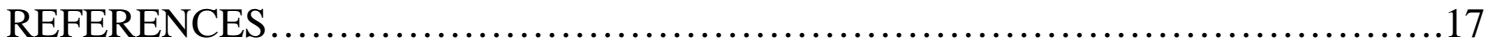

\section{LIST OF GRAPHICAL MATERIALS}

Fig. 1. Electron beam evaporator used for carbide synthesis.

Fig. 2. Example powder diffraction pattern from cubic-stabilized lanthanum yttrium carbide.

Fig. 3. Carbon-12 SIMS profile with calculated diffusion curve (dotted) for lanthanum erbium carbide at $850^{\circ} \mathrm{C}$.

Fig. 4. The carbon diffusion coefficients for four ionic carbides at three different temperatures. 


\section{INTRODUCTION}

The crystal structure of a solid has a profound impact on both its ionic and electronic conductivity. There are approximately twenty common, or prototype, crystal structures and many less common structures that have the potential to show superionic conductivities. Despite the large variety of potential structures, high ionic conductivity has been found in only a few.

Ionic conduction does not lead to high conductivities in most ionic or covalent solids. Atoms tend to be fixed in their lattice sites and move via lattice defects. Only at higher temperatures does ionic conduction occur to any appreciable amount in most solids. This result is due to the lattice defects produced at high temperatures. $\mathrm{NaCl}$ for example, has an ionic conductivity of $\sim 10^{-3} \mathrm{ohm}^{-1} \mathrm{~cm}^{-1}$ at $800^{\circ} \mathrm{C}$, just below its melting point. At room temperature $\mathrm{NaCl}$ has a conductivity of $\sim 10^{-12} \mathrm{ohm}^{-1} \mathrm{~cm}^{-1}$. In contrast, superionic conductors have high ionic conductivity even at temperatures well below their melting point (1). The conductivity for $\mathrm{Na}^{+}$ions through $\mathrm{B}$-alumina, for example, is $10^{-3}$ $\mathrm{ohm}^{-1} \mathrm{~cm}^{-1}$ at $25^{\circ} \mathrm{C}$, and is in the conductivity range for strong liquid electrolytes.

One important factor for good ionic conduction is a structure permeated by open channels, preferably in three dimensions, in which mobile ions can migrate. Open ionconduction channels may be generated in close packed structures by two types of interstitial sites, tetragonal and octahedral. Three atoms in one layer are needed to form the base of a tetragonal site together with one atom in the layer above or below to form the apex of the resulting tetrahedron. In contrast, an octahedral site is formed by six atoms in an octagonal configuration.

In the majority of crystal structures the larger, octahedral site is at least partially occupied. However, this is not true for antifluorite structures. The unoccupied octahedral sites in antifluorite structures provide large open spaces in which ions may move and a number of antifluorite compounds show superionic conductivity.

The antifluorite structure is composed of anions in a face centered cubic arrangement, with cations fully occupying the tetragonal interstitial sites. The prefix "anti" delineates it from its prototype crystal structure fluorite, named after the naturally occurring mineral $\mathrm{CaF}_{2}$. The fluorite structure is geometrically identical to the antifluorite structure, except that in it the cations form the cubic close packed structure, and the anions occupy the tetragonal interstitial sites.

The cation close packing of the fluorite structure is unusual because in general anions are larger than cations. In the fluorite structure the larger anions reside in the small interstitial sites rather than in the close packed structure. The anions are even further crowded, since in fluorite they are forced to occupy all the tetrahedral sites, instead of larger octahedral sites, to maintain electrical neutrality. This expansion creates larger passageways between interstitial sites for the migration of ions. 


\section{Existing lonic Conductors}

The ionic conductivity of the fluorite structure was discovered in the early 1800's by Michael Faraday, who studied the compound $\mathrm{PbF}_{2}$. In 1899 the first practical use for the fluorite structure as an ionic conductor was proposed by Nernst, who studied zirconia, $\mathrm{ZrO}_{2}$. Yttria-stabilized zirconia is in use now as the oxygen-ion superionic transport membrane material for a large number of fuel cells.

Another highly studied solid electrolyte is B-alumina, which exhibits very high $\mathrm{Na}^{+}$conductivity. The discovery of superionic conductivity in this compound led to an intense effort to develop high energy batteries using B-alumina as a solid electrolyte for sodium-sulfur batteries. The ideal formula for B-alumina derived from the crystal structure is $\mathrm{Na}_{2} \mathrm{O}-11\left(\mathrm{Al}_{2} \mathrm{O}_{3}\right)$, also written as $\mathrm{NaAl}_{11} \mathrm{O}_{17}$. To date, these efforts have failed due to the tendency of this material to short-circuit via metallic sodium paths that develop along grain boundaries.

No superionic conductor for carbon ions is yet known. The first requirement of any potential ionic carbon-ion conductor is that its carbon bonds have a strongly ionic character. Application of Pauling's rule for bond ionicity (2) shows that the lanthanide carbides have a bond ionicity which is comparable to the ionicity of the bonding in zirconium oxide, which is the superionic oxygen conductor of choice for carbon-ion based fuel cells. This work is aimed at the determination of the carbon-ion mobility of lanthanium carbides, which have been stabilized in the cubic structure that is the equivalent of the zirconia that has been stabilized in the cubic structure by yttria. In the Executive Summary, the scientific basis for the development of superionic carbon-ion conductors is outlined.

\section{EXECUTIVE SUMMARY}

Solid ionic electrolytes are a major factor in fuel cell development, but only a few compounds are known to be superionic. The fluorite structure, in particular, has been the basis for several superionic conductors of $\mathrm{F}^{-}, \mathrm{I}^{-}$, and $\mathrm{O}^{2-}$ ions.

Rare earth carbides are one of the few classes of carbon compounds which have a high degree of carbon-bond ionicity, and most carbides are covalently bonded. Additionally carbides of the form $\mathrm{LnC}_{2}$ (where $\mathrm{Ln}$ refers to any element of the lanthanide series) and have the fluorite structure when they are above their transition temperatures. These transition temperatures range from $350{ }^{\circ} \mathrm{C}\left(\mathrm{EuC}_{2}\right)$ to $1450{ }^{\circ} \mathrm{C}$ $\left(\mathrm{LuC}_{2}\right)$. The carbon ions in these compounds reside as anions in tetragonal positions equivalent to the positions of the mobile ions, $\mathrm{F}^{-}$and $\mathrm{O}^{2-}$ in the known superionic conductors $\mathrm{CaF}_{2}$ and $\mathrm{Zr}_{0.8} \mathrm{Y}_{0.2} \mathrm{O}_{2}$. These cubic lanthanide carbide compounds are thus potential candidates for ionic conductors of carbon. The discovery of a material with high carbon ion conductivity would be a major scientific advance, opening the possibility of an entirely new class of fuel cells that could convert carbon directly to $\mathrm{CO} / \mathrm{CO}_{2}$ and produce electric power without combustion. However, the first step must be to stabilize the fluorite structure.

In order to stabilize the cubic fluorite structure to low temperatures, a mixture of two different lanthanide dicarbides must be formed. The lanthanide carbide set, having a stabilized fluorite structure, that has been evaluated consists of $\mathrm{La}_{0.5} \mathrm{Er}_{0.5} \mathrm{C}_{2}, \mathrm{Ce}_{0.5} \mathrm{Er}_{0.5} \mathrm{C}_{2}$, and $\mathrm{La}_{0.5} \mathrm{Y}_{0.5} \mathrm{C}_{2}$, and $\mathrm{Ce}_{0.5} \mathrm{Tm}_{0.5} \mathrm{C}_{2}$ modified by the addition of 5 wt $\% \mathrm{BeC}_{2}$. The 
preparation of the lanthanum carbide $\mathrm{CeYbC}_{2}$ proved to be impossible due to the high vapor pressure of the compound $\mathrm{Yb}_{2} \mathrm{O}_{3}$. This precursor compound was found to vaporize completely before a reaction temperature high enough to form the carbide phase could be reached.

These hanthanide dicarbides have been synthesized by using our newly developed technique for reacting mixtures of $\mathrm{Ln}_{2} \mathrm{O}_{3}$ and amorphous ${ }^{13} \mathrm{C}$ under vacuum at high temperatures $\left(>1600{ }^{\circ} \mathrm{C}\right)$. Powder $\mathrm{x}$-ray diffraction was used to confirm the crystal structure of the synthesized compounds. Semi-thick coatings $(>10 \mu \mathrm{m})$ of ${ }^{12} \mathrm{C}$ were deposited on these samples using the arc-evaporation of graphite electrodes. The coated samples were heated at $850{ }^{\circ} \mathrm{C}, 950{ }^{\circ} \mathrm{C}$, and $1150{ }^{\circ} \mathrm{C}$ in a custom-built high vacuum furnace to allow the ${ }^{12} \mathrm{C}$ to diffuse into the bulk ${ }^{13} \mathrm{C}$ sample. Using secondary ion mass spectrometers(SIMS) facilities at North Carolina State University, the concentration of ${ }^{12} \mathrm{C}$ and ${ }^{13} \mathrm{C}$ profiles in bulk potential superionic carbides have been measured. These measurements were then used to determine a diffusion coefficient for carbon in the carbides as a function of temperature.

The diffusion coefficients for three different lanthanum carbides, including the effect of $\mathrm{Be}_{2} \mathrm{C}$ additions on the carbon diffusion rate as measured for $\mathrm{CeTmC}_{2} \cdot \mathrm{Be}_{2} \mathrm{C}$, since it has the antifluorite structure, offers the possibility of increasing the diffusion rate of carbon by disruption of the carbon ion sublattice. Diffusion rates have been measured for temperatures over the range $1150^{\circ} \mathrm{C}$ to $850^{\circ} \mathrm{C}$. These coefficients were found to range from approximately $2.0 \cdot 10^{-13} \mathrm{~cm}^{2} / \mathrm{sec}$ at $850{ }^{\circ} \mathrm{C}$ to $1.7 \cdot 10^{-12} \mathrm{~cm}^{2} / \mathrm{sec}$ at $1150{ }^{\circ} \mathrm{C}$. To our knowledge, these data represent the first time that diffusion coefficients for these materials have ever been measured. However, these values are not in the range of superionic conductors $\left(10^{-4}-10^{-7} \mathrm{~cm}^{2} / \mathrm{sec}\right)$, and additional compounds and modified compounds will have to be produced and tested if superionic membrane materials for carbon ions are to be successfully developed.

\section{EXPERIMENTAL}

The straightforward synthesis of lanthanide dicarbides directly from these elements proved elusive. An alternative method for formation of these dicarbides was developed via the combination of lanthanide oxides, instead of the lanthanide metal with carbon powder to form the lanthanide carbides:

$$
2 \mathrm{Ln}_{2} \mathrm{O}_{3}+11 \mathrm{C} \rightarrow 4 \mathrm{LnC}_{2}+3 \mathrm{CO}_{2}
$$

Examination of this reaction reveals the possibility that the gaseous reaction product is not carbon dioxide, since at elevated temperatures, carbon monoxide becomes the more thermodynamically stable carbon/oxygen reaction product. If carbon monoxide is the gaseous product, then the amount of required carbon changes. The revised equation will then be:

$$
\mathrm{Ln}_{2} \mathrm{O}_{3}+7 \mathrm{C} \rightarrow 2 \mathrm{LnC}_{2}+3 \mathrm{CO}
$$

Samples were heated using a high temperature vacuum induction furnace. The reaction was carried out until no more out-gassing of $\mathrm{CO}$ or $\mathrm{CO}_{2}$ was seen. Each sample was then heated to $>2000{ }^{\circ} \mathrm{C}$ in order to melt it into a homogenous pellet. This approach had several advantages over the direct reaction of lanthanid e metal with carbon powder. The use of lanthanide oxide powders was especially attractive because of the ease of 
control over stoichiometry. In addition, the lanthanide oxides are much cheaper and easier to store than elemental lanthanum because they are not reactive with the atmosphere.

\section{Hybrid Arc-Melt Furnace Technique}

Because of the extremely high temperatures required, a hybrid technique was also developed using an arc-melt furnace. Such furnaces have the advantage of being able to attain extreme temperatures and do not require a crucible. The arc melt furnace could be used to heat a pressed oxide/carbon pellet. By combining the oxide method with the arc melt furnace, a quick and effective technique for sample preparation was developed.

Cerium (IV) and Erbium (III) 99.9\% oxides and graphite were purchased from Johnson Matthey-Alfa in <20 micron powdered form. The samples were prepared by mixing two lanthanide oxides and graphite powder and then pressing the mixture into a pellet. The graphite/la nthanide oxide powder was pressed into $0.953 \mathrm{~cm}$ diameter pellets using a die built by the Duke Mechanical Engineering machine shop and a 32-ton capacity hydraulic press. The samples were pressed at 100,000 psi for a minimum of twenty minutes. Samples that were pressed for less than twenty minutes, or at lower pressures, tended to separate into several layers, making handling and heating very difficult. Samples that had been pressed for sufficient time were hard and dense with considerable mechanical strength. Most samples weighed approximately 1.5 grams and were about $4 \mathrm{~mm}$ thick. This thickness was approximately the maximum thickness that could be easily pressed into pellets. Thicker samples often broke, because friction from the die walls caused anisotropic pressures within the pellet.

The formed oxide/graphite pellet was placed in the arc-melt furnace, consisting of a water-cooled vacuum chamber in which an arc is stuck between a moveable carbon rod and the copper base of the furnace. The pellet was placed in a small indentation in the copper base, so no crucible was needed. Since the base is water-cooled, the copper does not melt or contaminate the sample. The chamber was initially evacuated to remove any oxygen and water vapor, using a rotary vane pump. It was then flushed with argon, and pumped down again. An arc was initially struck between the moveable carbon rod and a small titanium gettering pellet. The titanium was heated until it was completely melted to lower the level of remaining oxygen and other contaminants in the chamber. The arc was then carefully brought in close proximity to the oxide pellet by positioning the moveable carbon rod. The pressure, current, and position of the arc were continually adjusted to allow for a smooth uniform reaction of the sample. As the pellet was heated by the arc, the carbon began to react with the oxide; this reaction could be visually observed by the gradual formation of liquid carbide on the sample surface and the outgassing of $\mathrm{CO} / \mathrm{CO}_{2}$.

If the heating rate is too high, the carbon and oxide react quickly, and rapid outgassing causes the sample to splatter. It was therefore important to heat the sample to a high enough temperature to obtain a reaction, but slow enough to allow the $\mathrm{CO} / \mathrm{CO}_{2}$ to escape. This was a rather delicate procedure and had to be learned through extensive practice. Even with careful heating, the sample reacted very quickly, and some of the carbide is always lost to splattering. Because of the erratic behavior of the arc, the 
heating rates and the reaction times varied. However, both were generally very rapid. The reaction times were typically about five to ten minutes.

After the sample had been completely reacted, the sample was cooled for five minutes before the furnace was opened, and the sample was flipped over and reheated. The reheating ensures thorough heating, mixing, and homogeneity throughout the sample. The lanthanide carbide sample was generally gold colored, button shaped, and approximately $6 \mathrm{~mm}$ in diameter by $3 \mathrm{~mm}$ thick. A typical reaction yielded approximately one gram of carbide. This yield corresponds to a recovery of about $80 \%$ of the starting materials. If none of the reactants were lost and the reaction went to completion, 1.2 grams of carbide should have been produced. The losses are believed to come from the splattering of the sample caused by the production of carbon monoxide and vaporization of the sample.

The produced lanthanide carbides react rapidly with $\mathrm{H}_{2} \mathrm{O}$ vapor in the air and must be quickly removed from the furnace and transported to a dry-box or desiccator for storage and x-ray or SIMS preparation to minimize sample degradation.

\section{Formation of Stable Ln-Oxide/Carbon Pellets}

Forming the lanthanide oxide/carbon pellets was very difficult. Several experiments were conducted solely on forming mechanically stable pellets. The key discovery that allowed successful formation of stable pellets was the recognition of the importance of the type of carbon used. For the first several experiments carbon black and amorphous carbon were used. Despite several attempts, neither of these types of carbon yielded stable pellets. When graphite was used, mechanically strong pellets could be formed over a wide variety of pressures. However, non-graphite samples, ranging in size from $0.635-1.27 \mathrm{~cm}$, pressed at pressures up to $150,000 \mathrm{psi}$, failed to yield anything except extremely weak pellets that were too fragile to be manipulated.

It is very beneficial to have the lanthanide oxide and carbon in a solid pellet. The formation of a mechanically stable oxide and carbon pellet could only be obtained using graphitic carbon. This posed a significant problem, since the commercially available ${ }^{13} \mathrm{C}$ comes only in amorphous form. When in a pellet form, there is much greater surface contact between the carbon and the oxide. This is important for the chemical reaction to form the carbide from the oxide, because the greater the surface contact, the more easily the reaction occurs. With low surface contact the reaction had to be run at a higher temperature and for a longer duration in order to ensure a complete reaction. Another advantage of the pellet form is that the lanthanide oxide carbon mixture has less contact with the walls of the heated carbon crucible. This allows the lanthanide carbide to be more easily separated from the graphite crucible at the end of the reaction and also helps to reduce the amount of higher carbides $\left(\operatorname{Ln}_{2} \mathrm{C}_{3}\right)$ that are reportedly formed when there is an excess of carbon.

Some initial investigations were done to explore the conversion of amorphous carbon to graphitic carbon. With the equipment available, this would have been a daunting task. The basic principle in forming graphitic carbon is to heat the amorphous carbon to extreme temperatures $\left(>2500^{\circ} \mathrm{C}\right)$ for long periods of time, sometimes weeks. This would have been extremely time consuming and would have pushed our equipment far beyond its limits. Because of the difficulties associated with converting the carbon 13 
to graphitic carbon 13, it was decided that effort should be directed to forming a mechanically stable pellet.

The first attempt at making a pellet was to press the oxide/carbon powder into a solid. A standard die was used in the 32 ton press for preparing pellets ranging in diameter from $6.35 \mathrm{~mm}$ to $12.70 \mathrm{~mm}$. The pressure was also varied up to 150,000 psi. Special carbide platens had to be made to withstand these high pressures.

Heating of the die was also tried. The entire die was heated up to $200{ }^{\circ} \mathrm{C}$ by a resistive heater during pressing. After many iterations at different pressures, temperatures, and durations, no mechanically strong pellet was obtained. The best results were obtained from a $6.35 \mathrm{~mm}$ die at $150,000 \mathrm{psi}$; however the pellet produced was not strong enough to be handled and placed into the heating crucible. However, there was a noticeable increase in the density of the powder after pressing: this pressed powder occupied much less volume then the unpressed powder. Although this was not as successful as forming a pellet, it was still advantageous. Increased density corresponds to greater surface contact between the lanthanide oxide and the carbon, thus making the chemical conversion of lanthanide oxide to lanthanide carbon easier.

A third and final technique for lanthanide carbide production was developed using an electron beam evaporator. The electron beam evaporator turned out to be ideal for lanthanide production. Electron beam guns are capable of reaching several thousands of degrees, and could easily reach the temperatures needed to react the lanthanide oxides to form the dicarbides. The electron beam evaporator consists of a single electron gun mounted on the bottom of a high vacuum chamber, as shown below

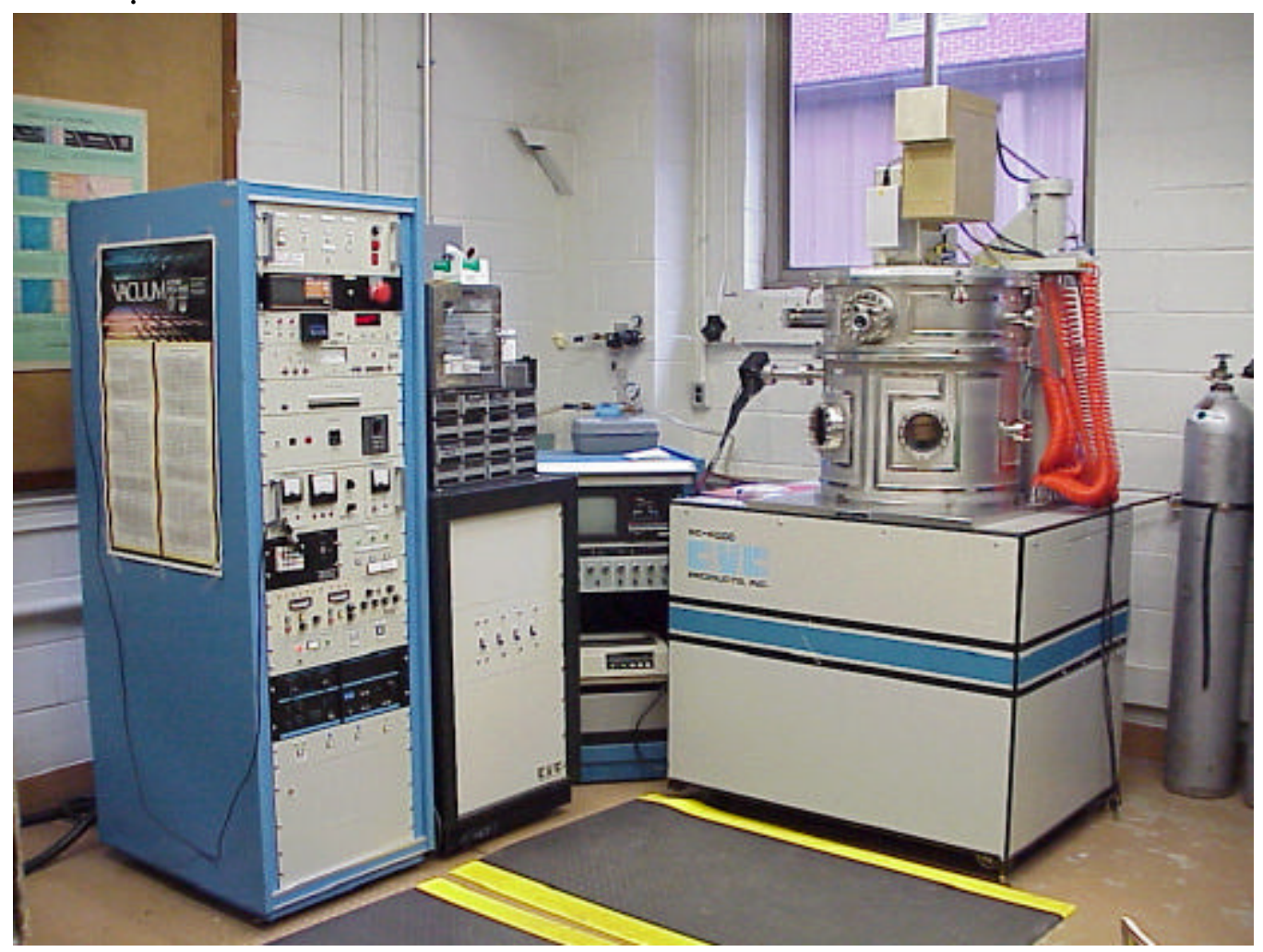

Figure 1. Electron Beam Evaporator Used For Carbide Synthesis 
Using a cryo-pump, the chamber is capable of reaching a high vacuum of $5 \cdot 10^{-8}$ torr. This is ideal for lanthanide carbide production because there is virtually no residual oxygen or water vapor to react with the carbide and degrade the integrity of the sample. Contamination by oxygen and moisture have been a concern in previous synthesis techniques, which obtained relatively poor vacuum $\left(1 \bullet 10^{-3}\right.$ torr $)$ using only a mechanical pump.

In the EBE synthesis process, lanthanide oxide/carbon powder was placed in a special graphite crucible. A tightly fitted, vented, graphite lid was then placed on the crucible covering the powder. The vent was a small hole in the side of the graphite lid which allowed the $\mathrm{CO} / \mathrm{CO}_{2}$ produced in the reaction to escape. The crucible was placed into the electron beam evaporator, and the chamber was pumped down to a pressure less than $1 \cdot 10^{-6}$ torr. The electron beam was then energized and heated the crucible. The electron beam was adjusted so that it rastered over the entire lid of the crucible because a stationary electron beam would hit the crucible lid in a concentrated area. This concentrated, stationary beam would vaporize a hole directly though the crucible lid, the lanthanide carbide, and out the crucible base. This result was discovered during a test run to determine the ability of the electron beam to heat graphite. Rastering was used in all experiments after this discovery.

Even with rastering a considerable amount of carbon was vaporized from the lid surface over the duration of a complete synthesis. In some cases, the electron beam vaporized through the graphite lid. This was not desirable because the electron beam contacted the carbide sample directly causing decomposition and scattering of the carbide. This burn-through problem was solved by adjusting the thickness of the graphite.

The electron beam current was gradually increased until the crucible was red hot. At this point a quadrapole gas analyzer was turned on. This device was extremely useful in the synthesis of the lanthanide dicarbides, because it determined the type and the amount of gases in the vacuum chamber. This was useful since the reaction of the lanthanide oxide and carbon produced $\mathrm{CO}$ and/or $\mathrm{CO}_{2}$ gas. Because the sample crucible was heated from the top, a temperature gradient was created in the sample. At lower electron beam currents only the top portion of the sample reached the extreme temperatures needed for the lanthanide oxides and carbon to react. If a higher electron beam current was immediately used to bring the entire sample up to the reaction temperature, the reaction was too fast for the vacuum pump to maintain the low pressure in the chamber needed to sustain the electron beam. Therefore, the electron beam current had to be slowly increased for the duration of the reaction. The reaction was assumed to be complete when increasing the electron beam current no longer increased the $\mathrm{CO} / \mathrm{CO}_{2}$ pressure.

This current adjustment was initially done by hand, increasing the current to maintain a pressure of approximately $9.0 \cdot 10^{-6}$ torr inside the chamber. This was a long and tedious process. The reaction times were very long, generally greater than twelve hours, and the pressure was very sensitive to small changes in the electron beam current. Because of this difficulty, a programmable PID controller was installed to adjust the beam current. By setting a desired pressure on the controller, it continuously adjusted the electron beam current to maintain the set pressure. The controller read a 0-10 V signal from the pressure gauge that corresponds to the chamber pressure. Using a proportional 
and integral control, a 0-20 mA signal was outputted from the controller. This 0-20 mA signal was converted into a $0-10 \mathrm{~V}$ signal that controlled the electron beam current.

Because the PID controller was continuously adjusting the beam current, the reaction times were much quicker than when it was adjusted by hand. Instead of taking in excess of twelve hours, the reaction times were reduced to eight hours or less. Visual inspection of the sample showed a brilliant, porous, golden material, characteristic of the lanthanide carbides. Further analysis by x-ray diffraction revealed that the sample was indeed lanthanide dicarbide. This method had the same porosity problem as the resistive heating technique, but the problem could be overcome by melting the porous pellet in the same crucible by further increasing the electron beam power or using the arc-melt furnace. At the temperature needed to melt the sample the electron beam current was approximately 0.2 amps. The maximum current for the electron beam gun is $0.8 \mathrm{amps}$, so insufficient heating is not a problem in the electron beam technique as it was in the resistive heating.

Samples of $\mathrm{LaC}_{2}, \mathrm{ErC}_{2}, \mathrm{CeC}_{2}, \mathrm{YC}_{2}, \mathrm{La}_{0.5} \mathrm{Er}_{0.5} \mathrm{C}_{2}, \mathrm{Ce}_{0.5} \mathrm{Er}_{0.5} \mathrm{C}_{2}$, and $\mathrm{Y}_{0.5} \mathrm{Er}_{0.5} \mathrm{C}_{2}$ have been produced using the electron beam technique. This technique, as we have developed it, has proven to be very reliable and repeatable

\section{RESULTS AND DISCUSSION}

X-ray diffraction was used to determine the crystal structure of the lanthanide carbides dicarbides as synthesized by the procedure described in detail in the Experimental section. Crystal structure verification was needed after synthesis to ensure the correct carbide with the fluorite structure had been produced. Dicarbides are only fluorite-structured when two different carbides are alloyed together in correct proportions.

Using a Phillips x-ray diffractometer, theta/2-theta coupled powder xray scans were obtained. $\mathrm{Cu}$ Ka radiation was determined to be the best overall choice, because of better peak resolution. These scans provided lattice parameters as well as structural information. A minimum of two scans were taken of every sample. First a quick scan over a large 2-theta range was taken, followed by a much slower, more detailed scan of all of the peaks found in the first scan. By these means the room temperature cubic crystal structure of these materials was confirmed. An example x-ray diffraction pattern confirming the cubic crystal structure is shown in Figure 2.

Using the raw data from the SIMS experiments the diffusion coefficients were determined for each sample. The collected concentration versus depth profile from the SIMS measurements needed to be analyzed to determine the diffusion coefficient. Since the thick carbon coating applied to the samples did not totally diffuse into the bulk. the diffusion was modeled as a thick surface $\mathrm{C}^{12}$ film diffusing into a semi-infinite solid. The diffusion equation for this case is (3):

$$
c(x, t)=\frac{c^{\prime}-c_{o}}{2}\left[1-\operatorname{erf}\left(\frac{x}{2 \sqrt{D t}}\right)\right]+c_{o}
$$

where c' is the concentration of the film, $c_{o}$ is the concentration of the sample, $x$ is the depth from the interface of the film and sample, $D$ is the diffusion coefficient, and $t$ is the anneal time. 
Using this equation and Matlab ${ }^{\circledR}$ a computer program was written to analyze the raw SIMS data and compute the diffusion coefficients. This program uses a least-squares fitting routine to determine the diffusion coefficient. In general, the diffusion curves fit well with the collected data. Fig. 4 shows the collection of the three different lanthanide carbide diffusion measurements determined in this way and includes also aluminum carbide, $\mathrm{Al}_{4} \mathrm{C}_{3}$, which was also prepared and tested. A straight line can be fit through this data. From the slope of this line the activation energy for the carbon can be calculated with the use of the Arrhenious equation. The calculated activation energy for lanthanumerbium carbide and aluminum carbide was $95 \mathrm{~kJ} / \mathrm{mol}$, and $135 \mathrm{~kJ} / \mathrm{mol}$ respectively. The resulting diffusion constants as measured were all less than $10^{-11} \mathrm{~cm}^{2} / \mathrm{sec}$, and therefore these compounds are not superionic. However, there are a large number of potentially superionic pseudobinary lanthanide compounds, and the discovery of a superionic conductor for carbon ions will require testing numerous lanthanide carbide pseudobinary combinations, including the addition as dopants of alternative carbides that have the ability to produce vacancies on the carbon-ion sublattice to increase diffusion rates. 


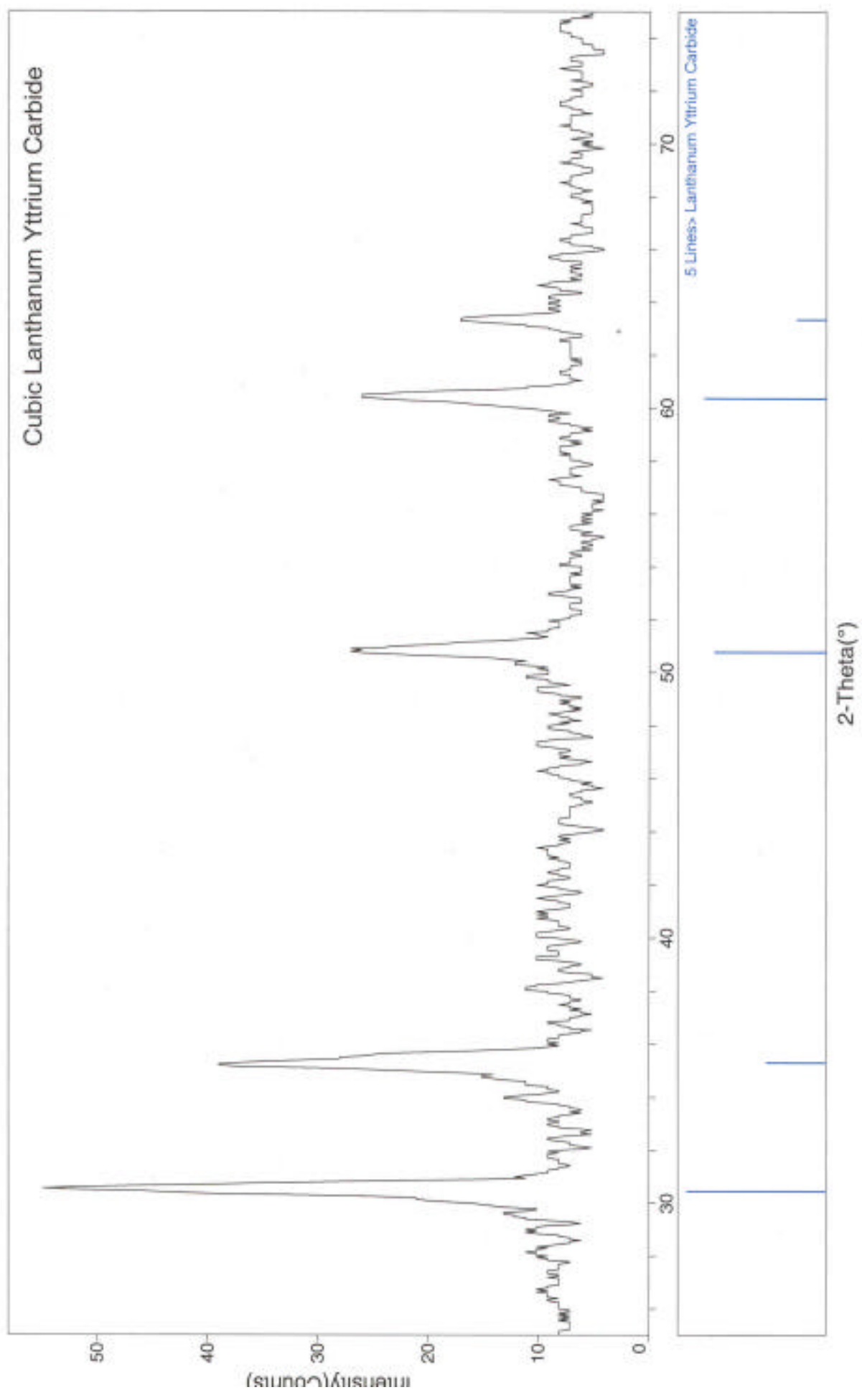

Figure 2. Example powder diffraction pattern from cubic-stabilized lanthanum yttrium carbide 


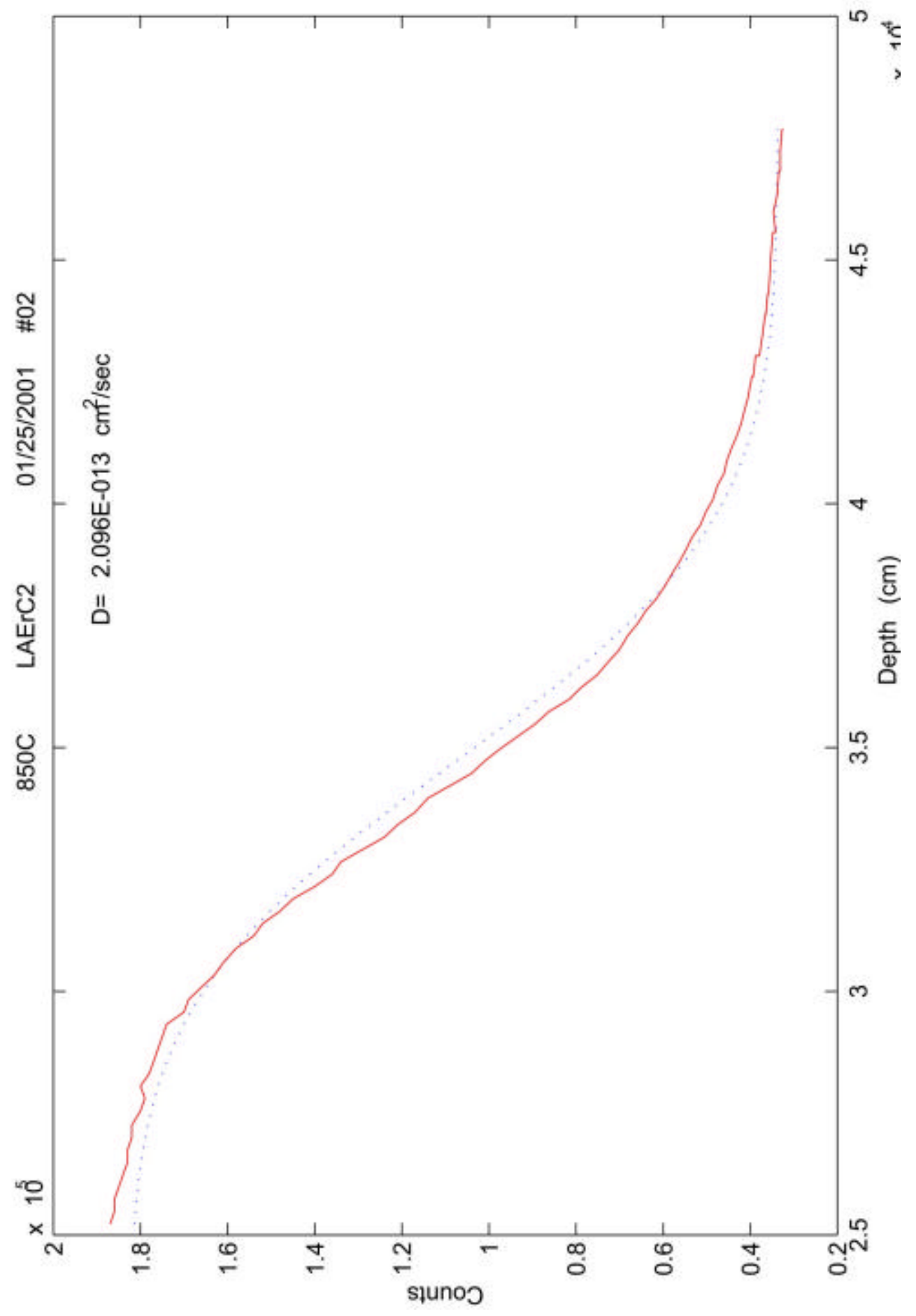

Figure 3. Carbon-12 SIMS profile with calculated diffusion curve (dotted) for lanthanum erbium carbide $\left(850{ }^{\circ} \mathrm{C}\right)$. 


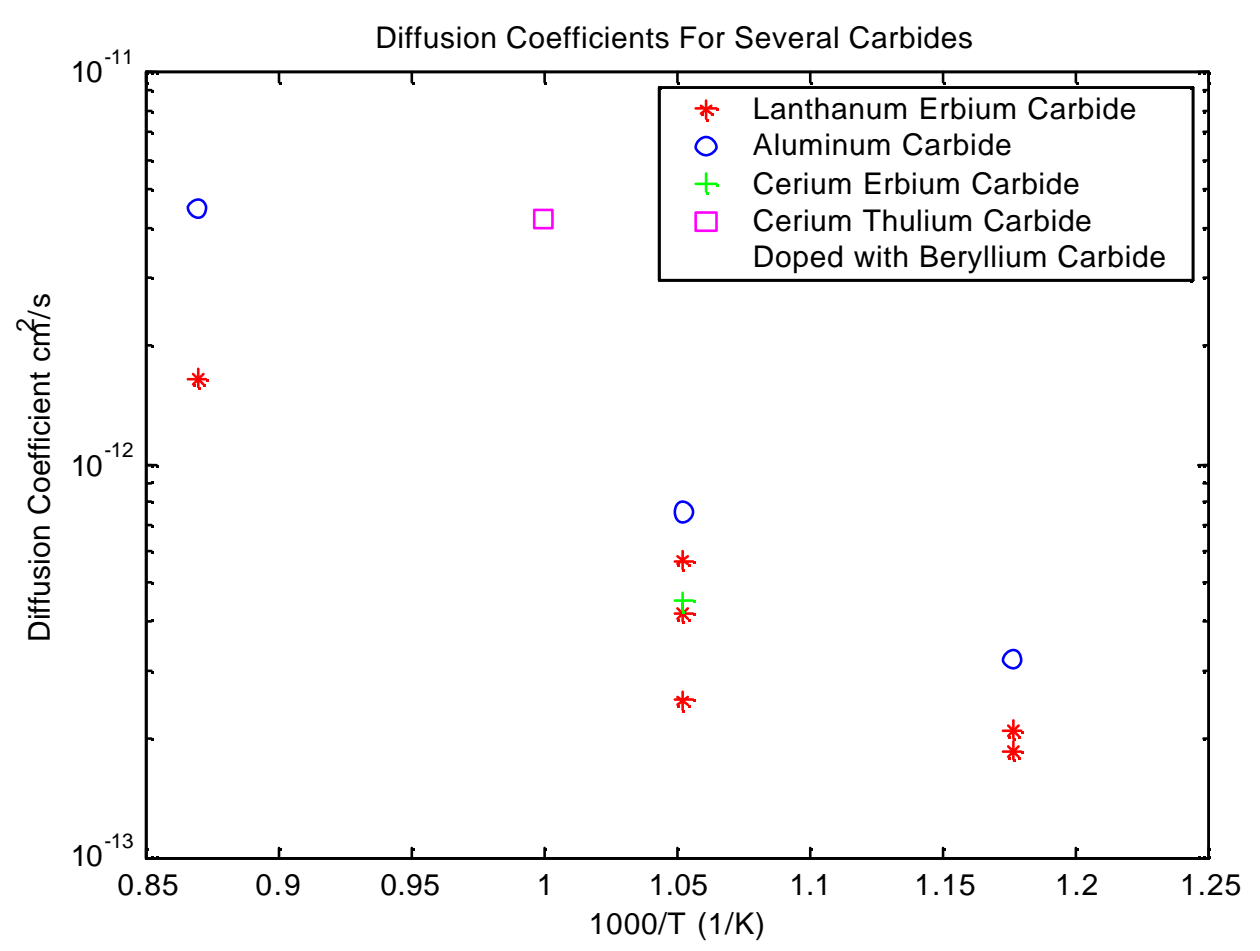

Figure 4. The diffusion coefficients of four ionic carbides at three different temperatures

\section{Conclusion}

The objective of this research was the investigation of ionic lanthanide carbides, as well as ionic lanthanide carbide pseudo-binary solid solutions, both of which have a high carbon-metal bond ionicity, as possible superionic carbide-ion conductors. Carbonconsuming fuel cells have many potential advantages in power generation from coal, including increased efficiency and reduced pollution. A carbon-ion superionic membrane material would enable an entirely new class of carbon fuel cell to be developed, one that would use coal directly as the fuel source, without any intervening combustion process. However, a suitable superionic membrane material for carbon ions is not yet known.

Mixed lanthanide carbides have been synthesized using a new electron beam technique. Mixed lanthanide carbides produced by this method were stabilized in the fluorite structure at room temperature. The carbon diffusion rates in $\mathrm{La}_{0.5} \mathrm{Er}_{0.5} \mathrm{C}_{2}, \mathrm{Ce}_{0.5} \mathrm{Er}_{0.5} \mathrm{C}_{2}$, and $\mathrm{La}_{0.5} \mathrm{Y}_{0.5} \mathrm{C}_{2}$, and $\mathrm{Ce}_{0.5} \mathrm{Tm}_{0.5} \mathrm{C}_{2}$ modified by the addition of 5 wt $\% \mathrm{Be}_{2} \mathrm{C}$ have been determined by means of a $\mathrm{C}^{12} / \mathrm{C}^{13}$ technique that has been developed for this purpose. Using secondary ion mass spectrometry (SIMS) the diffusion coefficient of carbon through the fluorite structured $\mathrm{La}_{0.5} \mathrm{Er}_{0.5} \mathrm{C}_{2}$ has been determined to be $2.0 \cdot 10^{-13}, 4.1 \cdot 10^{-13}$, and $1.7 \cdot 10^{-12} \mathrm{~cm}^{2} / \mathrm{sec}$ at $850{ }^{\circ} \mathrm{C}, 950{ }^{\circ} \mathrm{C}$ and $1150{ }^{\circ} \mathrm{C}$ respectively via the measured $\mathrm{C}^{12}$ gradients produced by heating these lanthanide carbides after they have been coated with a surface layer of $\mathrm{C}^{12}$. From these measurements the activation energy for carbon diffusion was calculated to be $95 \pm 20 \mathrm{~kJ} / \mathrm{mol}$. Since these diffusion constants are all less than $10^{-11} \mathrm{~cm}^{2} / \mathrm{sec}$, none of these compounds are superionic. However, there are a large number of potentially superionic pseudobinary lanthanide compounds. It appears that the 
discovery of a superionic conductor for carbon ions will require the testing of numerous lanthanide carbide pseudobinary combinations, as well as the addition of alternative carbides which have the ability to produce vacancies on the carbon-ion sublattice, to increase the carbon diffusion rate. The results described in this work represent the firstever measurements of carbon diffusion for these ionic carbide compounds, and are the initial step towards the development of a superionic carbon-ion conductor that would make possible a truly revolutionary fuelcell coal technology.

\section{REFERENCES}

1. S. Geller, Solid Electrolytes, Springer Verlag, Topics in Applied Physics Series, Berlin, 1977.

2. L. Pauling, The Nature of the Chemical Bond, $3^{\text {rd }}$ ed, Cornell University press, Ithica, NY, 1960.

3. P. Shewman, Diffusion in Solids, $2^{\text {nd }}$ ed. 1987, The Minerals, Metals and materials Society, Warrendale, PA. 